\title{
ON A GRAPH PACKING CONJECTURE BY BOLLOBÁS, ELDRIDGE AND CATLIN*
}

\author{
HEMANSHU KAUL, ALEXANDR KOSTOCHKA, GEXIN YU
}

\author{
Received February 19, 2006
}

\begin{abstract}
Two graphs $G_{1}$ and $G_{2}$ of order $n$ pack if there exist injective mappings of their vertex sets into $[n]$, such that the images of the edge sets are disjoint. In 1978, Bollobás and Eldridge, and independently Catlin, conjectured that if $\left(\Delta\left(G_{1}\right)+1\right)\left(\Delta\left(G_{2}\right)+1\right) \leq n+1$, then $G_{1}$ and $G_{2}$ pack. Towards this conjecture, we show that for $\Delta\left(G_{1}\right), \Delta\left(G_{2}\right) \geq 300$, if $\left(\Delta\left(G_{1}\right)+1\right)\left(\Delta\left(G_{2}\right)+1\right) \leq 0.6 n+1$, then $G_{1}$ and $G_{2}$ pack. This is also an improvement, for large maximum degrees, over the classical result by Sauer and Spencer that $G_{1}$ and $G_{2}$ pack if $\Delta\left(G_{1}\right) \Delta\left(G_{2}\right)<0.5 n$.
\end{abstract}

\section{Introduction}

Two $n$-vertex graphs $G_{1}$ and $G_{2}$ are said to pack if there exist injective mappings of their vertex sets onto $[n]=\{1, \ldots, n\}$ such that the images of the edge sets do not intersect. In other words, $G_{1}$ and $G_{2}$ pack if $G_{1}$ is isomorphic to a subgraph of the complement of $G_{2}$. This concept leads to a natural generalization of a number of problems in extremal graph theory, such as existence of a fixed subgraph, equitable colorings, and Turán-type problems.

The study of extremal problems on packings of graphs was started in the 1970s by Bollobás and Eldridge [3,4], Sauer and Spencer [13], and Catlin [6].

Mathematics Subject Classification (2000): 05C35, 05C70

* This work was supported in part by NSF grant DMS-0400498. The work of the second author was also partly supported by NSF grant DMS-0650784 and grant 05-01-00816 of the Russian Foundation for Basic Research. The work of the third author was supported in part by NSF grant DMS-0652306. 
(See the surveys by Wozniak [14] and Yap [15] for later developments in this field.) In particular, Sauer and Spencer [13] proved the following result.

Theorem 1 (Sauer and Spencer). Let $G_{1}$ and $G_{2}$ be $n$-vertex graphs with maximum degrees $\Delta_{1}$ and $\Delta_{2}$, respectively. If $2 \Delta_{1} \Delta_{2}<n$, then $G_{1}$ and $G_{2}$ pack.

Kaul and Kostochka [12] gave a characterization of the pairs $\left(G_{1}, G_{2}\right)$ of $n$-vertex graphs with $2 \Delta_{1} \Delta_{2}=n$ that do not pack.

The main conjecture in this area was posed in 1978 by Bollobás and Eldridge [4], and independently by Catlin [7].

Conjecture 1 (Bollobás, Eldridge, and Catlin). Let $G_{1}$ and $G_{2}$ be $n$ vertex graphs with maximum degrees $\Delta_{1}$ and $\Delta_{2}$. If $\left(\Delta_{1}+1\right)\left(\Delta_{2}+1\right) \leq n+1$, then there is a packing of $G_{1}$ and $G_{2}$.

If true, this conjecture would be sharp, and a considerable extension of the Hajnal-Szemerédi Theorem [10] on equitable colorings. The HajnalSzemerédi Theorem is a special case of the conjecture when $G_{2}$ is the disjoint union of cliques of the same size. This conjecture has been proved in the case $\Delta_{1} \leq 2$ by Aigner and Brandt [1] and Alon and Fisher (for sufficiently large $n$ ) [2], and in the case when $\Delta_{1}=3$ and $n$ is huge by Csaba, Shokoufandeh, and Szemerédi [8]. Bollobás, Kostochka and Nakprasit [5] proved a strengthening of the conjecture when $G_{1}$ is $d$-degenerate and $d<\Delta_{1} / 40$. Eaton [9] showed that under the given condition, there is a near-packing of degree 1 of $G_{1}$ and $G_{2}$, that is, an embedding of the two graphs into a common vertex set such that the maximum degree of the subgraph defined by the edges common to both copies is 1 .

In this paper, instead of proving the conjecture for another class of graphs we give a weaker bound for all graphs with high maximum degrees.

Problem 1. For a fixed $0 \leq \epsilon \leq 1$, and $\left|V\left(G_{1}\right)\right|=\left|V\left(G_{2}\right)\right|=n$ and

$$
\left(\Delta_{1}+1\right)\left(\Delta_{2}+1\right) \leq \frac{1}{2} n(1+\epsilon)+1 .
$$

Do $G_{1}$ and $G_{2}$ pack?

The case $\epsilon=0$ is essentially the Sauer-Spencer Theorem, while the case $\epsilon=1$ is the Bollobás-Eldridge-Catlin conjecture. We show that if $G_{1}$ and $G_{2}$ satisfy the above condition for $\epsilon=0.2$, and if $\Delta_{1}$ and $\Delta_{2}$ are relatively large, then $G_{1}$ and $G_{2}$ pack. 
Theorem 2. Let $G_{1}$ and $G_{2}$ be $n$-vertex graphs with maximum degrees $\Delta_{1}$ and $\Delta_{2}$, respectively. If $\Delta_{1}, \Delta_{2} \geq 300$ and

$$
\left(\Delta_{1}+1\right)\left(\Delta_{2}+1\right) \leq 0.6 n+1,
$$

then $G_{1}$ and $G_{2}$ pack.

This improves the bound of the Sauer-Spencer Theorem for large $\Delta_{1}$ and $\Delta_{2}$ and thus partially answers Problem 4.4 in [11].

In the next section, we give some definitions and prove a basic lemma that is heavily used later. In Section 3 we derive some structural properties of a hypothetical minimal counterexample to our theorem. In Section 4 we prove the theorem modulo the main lemma, Lemma 2. Lemma 2 is proved in the last two sections.

\section{Preliminaries}

Without loss of generality, we fix a graph $G_{1}$ with $V\left(G_{1}\right)=V_{1}=[n]$. Given $G_{2}$ with $V_{2}=V\left(G_{2}\right)$ and $\left|V_{2}\right|=n$, we will try to find an injective $f: V_{2} \rightarrow[n]$ extending to an embedding of $G_{2}$ in $\overline{G_{1}}$.

The result of each bijection $f: V_{2} \rightarrow[n]$ will be viewed as a (multi)graph $G$ with edges of two types. The vertex set is $[n]$, and two vertices $u_{1}$ and $u_{2}$ are connected by an edge in $E_{1}$ if $u_{1} u_{2} \in E\left(G_{1}\right)$ and by an edge in $E_{2}$ if $f^{-1}\left(u_{1}\right) f^{-1}\left(u_{2}\right) \in E\left(G_{2}\right)$. Each vertex $u \in V(G)$ has two kinds of neighborhoods: $N_{1}(u)=\left\{v \in V(G): u v \in E_{1}\right\}$ and $N_{2}(u)=\{v \in V(G): u v \in$ $\left.E_{2}\right\}$. Let $N(u)=N_{1}(u) \cup N_{2}(u)$ and $d_{1}(u)=\left|N_{1}(u)\right|$ and $d_{2}(u)=\left|N_{2}(u)\right|$.

For each such mapping $f$ and $i, j \in\{1,2\}$, a $(u, v ; i, j)$-link is a path of length two from $u \in V(G)$ to $v \in V(G)$ passing through some vertex $w \in V(G)$ such that $u w \in E_{i}$ and $w v \in E_{j}$. A link is a $(u, v ; i, j)$-link for some $u, v \in V(G)$ and $i, j \in\{1,2\}$ with $i \neq j$.

A $\left(u_{1}, u_{2}, \ldots, u_{k}\right)$-swap replaces a mapping $f$ with a mapping $f^{\prime}$ that differs from $f$ only in that $\left(f^{\prime}\right)^{-1}\left(u_{i}\right)=f^{-1}\left(u_{i-1}\right)$, for $1 \leq i \leq k$, where indices are treated modulo $k$.

The following lemma provides a basic tool that will be used repeatedly in the proof. It allows us to transform an embedding of $G_{2}$ by making 'vertex swaps' that do not increase the number of conflicting edges. In fact, often a good choice of the vertices to be swapped will lead to disappearing of conflicting edges. In the statement of the lemma, the indices sum modulo $k$.

Lemma 1. Let $G_{1}, G_{2}, f, G$, links and vertex swaps be defined as above. Let $u_{1}, \ldots, u_{k}$ be vertices of $G$. 
(1) Suppose that there are no $\left(u_{i}, u_{i+1} ; 2,1\right)$-links for any $i$. If there are no $1 \leq i<j \leq k$ such that $u_{i} u_{j} \in E\left(G_{2}\right)$ and $u_{i+1} u_{j+1} \in E\left(G_{1}\right)$, then a $\left(u_{1}, \ldots, u_{k}\right)$-swap does not create new conflicting edges.

(2) Suppose that there are no $\left(u_{i}, u_{i+1} ; 1,2\right)$-links for any $i$. If there are no $1 \leq i<j \leq k$ such that $u_{i} u_{j} \in E\left(G_{1}\right)$ and $u_{i+1} u_{j+1} \in E\left(G_{2}\right)$, then a $\left(u_{1}, u_{k}, \ldots, u_{2}\right)$-swap does not create new conflicting edges.

Proof. Assume that $G$ does contain a sequence $u_{1}, \ldots, u_{k}$ satisfying conditions in (1) of the lemma. Consider the mapping $f^{\prime}$ that differs from $f$ only in that $\left(f^{\prime}\right)^{-1}\left(u_{i}\right)=f^{-1}\left(u_{i+1}\right)$ for $i=1, \ldots, k$ (indices are taken modulo $k$ ). Let $G^{\prime}=G\left(f^{\prime}\right)$.

Since $G$ has no $\left(u_{i}, u_{i+1} ; 2,1\right)$-links for any $i$, no conflicting edge in $G^{\prime}$ connects the set $U=\left\{u_{1}, \ldots, u_{k}\right\}$ with $V\left(G^{\prime}\right)-U$. Because there are no $1 \leq i<j \leq k$ such that $u_{i} u_{j} \in E\left(G_{2}\right)$ and $u_{i+1} u_{j+1} \in E\left(G_{1}\right)$, no conflicting edge in $G^{\prime}$ appears inside $U$.

The proof for (2) is similar.

This lemma is a useful generalization of the idea of the proof of Theorem 1 [13] by Sauer and Spencer.

\section{Properties of hypothetical counterexamples}

Suppose that Theorem 2 does not hold. Then for some $n$ there is a critical pair $\left(G_{1}, G_{2}\right)$ of $n$-vertex graphs $G_{1}$ and $G_{2}$ satisfying the conditions of the theorem. By the choice of the pair $\left(G_{1}, G_{2}\right)$, there is a mapping $f: V\left(G_{2}\right) \rightarrow$ $[n]$ such that some edge $e=u^{*} v^{*}$ of the resulting multigraph $G$ is the only parallel edge in $G$.

Lemma 1 immediately yields that every vertex in $G$ is within distance two of $u^{*}$ :

Claim 1. For every vertex $v \in V(G)-\left\{u^{*}, v^{*}\right\}$, there is either a $\left(u^{*}, v ; 1,2\right)$ link or a $\left(u^{*}, v ; 2,1\right)$-link.

Proof. Suppose not. Then after a $\left(u^{*}, v\right)$-swap there are no parallel edges between $u^{*}$ and $v^{*}$, and by Lemma $1, G_{1}$ and $G_{2}$ pack.

Thus, $V(G)-\left\{v^{*}\right\}=N_{1}\left(N_{2}\left(u^{*}\right)\right) \cup N_{2}\left(N_{1}\left(u^{*}\right)\right)$. This allows us to partition $V(G)$ into meaningful subsets whose sizes can be estimated by counting arguments. Define $A_{1}=N_{2}\left(N_{1}\left(u^{*}\right)\right)-N\left(u^{*}\right)$ and $B_{1}=N_{1}\left(N_{2}\left(u^{*}\right)\right)-N\left(u^{*}\right)$. Let $A=A_{1}-B_{1}, B=B_{1}-A_{1}$ and $C=A_{1} \cap B_{1}$.

Let $\sigma=\Delta_{1}+\Delta_{2}$. By (1),

$$
\Delta_{1} \Delta_{2} \leq 0.6 n-\sigma .
$$


By (2), for $i=1,2$ we have $\Delta_{i} \leq(0.6 n-\sigma) / \Delta_{3-i}$. Since $\Delta_{1}, \Delta_{2} \geq 300$, we obtain that

$$
\Delta_{1}, \Delta_{2} \leq 0.002 n-2 \text { and } \sigma \leq 0.004 n-4 .
$$

Also by (2),

$$
\left|A_{1}\right|,\left|B_{1}\right| \leq \Delta_{1} \Delta_{2} \leq 0.6 n-\sigma .
$$

By Claim 1, $\left|A_{1} \cup B_{1}\right| \geq n-\sigma$.

It follows that

$$
|A|=\left|A_{1} \cup B_{1}\right|-\left|B_{1}\right| \geq(n-\sigma)-(0.6 n-\sigma)=0.4 n,
$$

and similarly,

$$
|B|=\left|A_{1} \cup B_{1}\right|-\left|A_{1}\right| \geq(n-\sigma)-(0.6 n-\sigma)=0.4 n .
$$

By Claim 1, $V(G)-\left\{v^{*}\right\}=N_{1}\left(N_{2}\left(u^{*}\right)\right) \cup N_{2}\left(N_{1}\left(u^{*}\right)\right)$. By definition, $N_{1}\left(N_{2}\left(u^{*}\right)\right) \cap N_{2}\left(N_{1}\left(u^{*}\right)\right)=C \cup\left\{u^{*}\right\}$, thus

$$
\begin{aligned}
|C| & =\left|N_{1}\left(N_{2}\left(u^{*}\right)\right)\right|+\left|N_{2}\left(N_{1}\left(u^{*}\right)\right)\right|-\left|N_{1}\left(N_{2}\left(u^{*}\right)\right) \cup N_{2}\left(N_{1}\left(u^{*}\right)\right)\right|-1 \\
& \leq 2 \Delta_{1} \Delta_{2}-(n-1)-1 \leq 1.2 n-2 \sigma-n=0.2 n-2 \sigma,
\end{aligned}
$$

it follows that

$$
|A|+|B| \geq n-\sigma-|C| \geq 0.8 n+\sigma .
$$

Lemma 1 allows us to make the following observation about vertices in $A$ and $B$.

Claim 2. For each $a \in A, b \in B$, there is an $(a, b ; 2,1)$-link.

Proof. Assume for a contradiction that for some $a \in A$ and $b \in B$, there is no $(a, b ; 2,1)$-link. Note that there is no $\left(b, u^{*} ; 2,1\right)$-link and no $\left(u^{*}, a ; 2,1\right)$-link. Also, $a u^{*} \notin E(G)$ and $b u^{*} \notin E(G)$, thus by Lemma 1, after an ( $\left.a, b, u^{*}\right)$-swap, $G_{1}$ and $G_{2}$ pack, a contradiction.

Next, we need to partition $C$ into more informative subsets. Let

$$
A^{\prime}=\left\{c \in C: \text { there exists } a \in A \text { such that } a c \notin E_{2},\right.
$$

$$
\text { and there is no }(a, c ; 2,1)-\operatorname{link}\}
$$

and

$$
B^{\prime}=\left\{c \in C: \text { there exists } b \in B \text { such that } b c \notin E_{1},\right.
$$

$$
\text { and there is no }(c, b ; 2,1) \text {-link }
$$

and let $C^{\prime}=C-A^{\prime}-B^{\prime}$.

$A^{\prime}$ and $B^{\prime}$ are defined in such a way that vertices in $A^{\prime}$ (respectively, $B^{\prime}$ ) behave 'similarly' to those in $A$ (respectively, $B$ ). 
Claim 3. $A^{\prime} \cap B^{\prime}=\emptyset$. Moreover, for each $c_{1} \in A^{\prime}$, and for each $b \in B$, there is a $\left(c_{1}, b ; 2,1\right)$-link; and for each $c_{2} \in B^{\prime}$ and for each $a \in A$, there is an $\left(a, c_{2} ; 2,1\right)$-link.

Proof. Suppose that $c \in A^{\prime} \cap B^{\prime}$. Then there exist $a \in A$ and $b \in B$ such that there is no $(c, b ; 2,1)$-link and there is no $(a, c ; 2,1)$-link and $c a \notin E_{2}$. Note that there is no $\left(u^{*}, a ; 2,1\right)$-link and there is no $\left(b, u^{*} ; 2,1\right)$-link, also, $u^{*} a, u^{*} b \notin E(G)$. Thus by Lemma 1 , after a $\left(u^{*}, a, c, b\right)$-swap, we get a packing of $G_{1}$ and $G_{2}$.

Moreover, let $c_{1} \in A^{\prime}$ and $b \in B$. Then by the above argument, $c_{1} \notin B^{\prime}$. Suppose for a contradiction that there is no $\left(c_{1}, b ; 2,1\right)$-link. Note that, since $c_{1} \notin B^{\prime}, b c_{1} \in E_{1}$. Also, by definition of $A^{\prime}$, there exists $a \in A$ such that there is no $\left(a, c_{1} ; 2,1\right)$-link and $c_{1} a \notin E_{2}$. As in the argument above, we can now apply Lemma 1 to get a contradiction through a $\left(u^{*}, a, c_{1}, b\right)$-swap.

The proof for $c_{2} \in B^{\prime}$ is similar.

\section{Proof of Theorem 2}

By symmetry, from now on we assume that $\left|B \cup B^{\prime}\right| \geq\left|A \cup A^{\prime}\right|$. Then by (7) we have

$$
\left|B \cup B^{\prime}\right| \geq 0.5\left(n-\sigma-\left|C^{\prime}\right|\right)
$$

The main idea of the proof is to count the number of pairs of vertices in $A \times B$ with a unique $(2,1)$-link between them, and get contradictory lower and upper bounds. If $\left|C^{\prime}\right|$ is 'small', we will use vertices from $B^{\prime}$ as well.

Let

$$
B_{0}=\left\{\begin{array}{l}
B \cup B^{\prime}, \text { if }\left|C^{\prime}\right| \leq \frac{1}{15} n, \\
B, \text { otherwise. }
\end{array}\right.
$$

and let $N$ denote the set of pairs $(a, b), a \in A, b \in B_{0}$ having a unique $(a, b ; 2,1)$-link.

Let $M$ be the set of central vertices lying on those unique links. The size of $M$ gives an upper bound on $|N|$ :

$$
|N| \leq|M| \Delta_{1} \Delta_{2}
$$

We will bound the size of $M$ by estimating its intersection with $B \cup B^{\prime}$ and $A \cup A^{\prime}$. 


\section{Lemma 2.}

(10)

$$
\left|M \cap\left(B \cup B^{\prime}\right)\right| \leq\left\{\begin{array}{l}
\Delta_{1} \Delta_{2}-|A|-\left|A^{\prime}\right|-\frac{1}{2}\left|C^{\prime}\right|+\frac{5 \sigma}{2}, \text { always } \\
\Delta_{1} \Delta_{2}-|A|-\left|A^{\prime}\right|-\left|C^{\prime}\right|+\frac{5 \sigma}{2}, \text { if }\left|B \cup B^{\prime}\right| \leq \frac{8}{15} n-\sigma .
\end{array}\right.
$$

Furthermore,

$$
\left|M \cap\left(A \cup A^{\prime}\right)\right| \leq \Delta_{1} \Delta_{2}-|B|-\left|B^{\prime}\right|-\left|C^{\prime}\right|+2.5 \sigma .
$$

This main lemma will be proved in the next two sections. By Claims 2 and 3 , it directly leads to the following bound on $|M|$ :

$$
\begin{aligned}
|M| & \leq\left|M \cap\left(B \cup B^{\prime}\right)\right|+\left|M \cap\left(A \cup A^{\prime}\right)\right|+\left|M \cap C^{\prime}\right|+\sigma \\
& \leq\left\{\begin{array}{l}
2 \Delta_{1} \Delta_{2}-n+\frac{1}{2}\left|C^{\prime}\right|+7 \sigma \leq 0.2 n+5 \sigma+\frac{1}{2}\left|C^{\prime}\right|, \text { always } \\
2 \Delta_{1} \Delta_{2}-n+7 \sigma \leq 0.2 n+5 \sigma, \text { if }\left|B \cup B^{\prime}\right| \leq \frac{8}{15} n-\sigma .
\end{array}\right.
\end{aligned}
$$

Next, we give a lower bound on $|N|$ by a counting argument.

Lemma 3. $|N| \geq 0.4 n\left(\left|B_{0}\right|-0.6 n+\left|B \cup B^{\prime} \cup C^{\prime}\right|\right)$.

Proof. Let $a \in A$. At most $\Delta_{1} \Delta_{2}(2,1)$-links start at $a$. By Claims 2 and 3, at least $\left(\left|B \cup B^{\prime}\right|-\left|B_{0}\right|\right)+\left(\left|C^{\prime}\right|-\sigma\right)$ of them land in $(B \cup C)-B_{0}$. It follows that for at most

$$
\Delta_{1} \Delta_{2}-\left(\left|B \cup B^{\prime}\right|-\left|B_{0}\right|\right)-\left|C^{\prime}\right|+\sigma-\left|B_{0}\right|=\Delta_{1} \Delta_{2}-\left|B \cup B^{\prime}\right|-\left|C^{\prime}\right|+\sigma
$$

vertices $b \in B_{0}$, an $(a, b ; 2,1)$-link is not unique.

There are $|A|\left|B_{0}\right|$ pairs $(a, b) \in A \times B_{0}$, and each of them is connected by a $(2,1)$-link. Therefore,

$$
\begin{aligned}
|N| & \geq|A|\left|B_{0}\right|-|A|\left(\Delta_{1} \Delta_{2}-\left|B \cup B^{\prime}\right|-\left|C^{\prime}\right|+\sigma\right) \\
& =|A|\left(\left|B_{0}\right|+\left|B \cup B^{\prime} \cup C^{\prime}\right|-\Delta_{1} \Delta_{2}-\sigma\right) \\
& \geq 0.4 n\left(\left|B_{0}\right|-0.6 n+\left|B \cup B^{\prime} \cup C^{\prime}\right|\right) .
\end{aligned}
$$

Now, using the lower and the upper bounds on $|N|$, we will get a contradiction. This would complete the proof of the theorem, modulo the proof of Lemma 2. 
Case 1. $\left|C^{\prime}\right| \geq \frac{1}{15} n$. Then $\left|B_{0}\right|=|B| \geq 0.4 n$ and $|N| \geq 0.4 n\left(-0.2 n+\mid B \cup B^{\prime} \cup\right.$ $\left.C^{\prime} \mid\right)$. Since $\left|C^{\prime}\right| \geq \frac{1}{15} n$, we have $\left|B \cup B^{\prime}\right| \leq(0.6 n-\sigma)-\left|C^{\prime}\right| \leq 8 / 15 n-\sigma$. Hence by (12), $|M| \leq 0.2 n+5 \sigma$. Combining with (8), we obtain

$$
\begin{aligned}
0.4 n\left(-0.2 n+\frac{1}{2}\left(n-\sigma-\left|C^{\prime}\right|\right)+\left|C^{\prime}\right|\right) \leq|N| & \leq|M| \Delta_{1} \Delta_{2} \\
& \leq(0.2 n+5 \sigma)(0.6 n-\sigma) .
\end{aligned}
$$

Since $\left|C^{\prime}\right| \geq \frac{1}{15} n$, we get

$$
0.2 n\left(\frac{2}{3} n-\sigma\right) \leq(0.2 n+5 \sigma)(0.6 n-\sigma) .
$$

Opening the parentheses, we come to

$$
\frac{n^{2}}{75}-3 n \sigma+5 \sigma^{2} \leq 0
$$

But this inequality does not hold for $0<\sigma<0.004 n$, a contradiction to (3).

Case 2. $\left|C^{\prime}\right|<\frac{1}{15} n$ and $\left|B \cup B^{\prime}\right| \leq \frac{8}{15} n-\sigma$. Then $B_{0}=B \cup B^{\prime}$ and by (8),

$$
|N| \geq 0.4 n\left(2\left|B \cup B^{\prime}\right|+\left|C^{\prime}\right|-0.6 n\right) \geq 0.4 n(0.4 n-\sigma) .
$$

Together with (12), we have

$$
0.4 n(0.4 n-\sigma) \leq|N| \leq|M| \Delta_{1} \Delta_{2} \leq(0.2 n+5 \sigma)(0.6 n-\sigma),
$$

which yields

$$
0.04 n^{2}-3.2 n \sigma+5 \sigma^{2} \leq 0 .
$$

Again, this inequality does not hold for $0<\sigma \leq 0.004 n$, a contradiction to (3). Case 3. $\left|C^{\prime}\right|<\frac{1}{15} n$ and $\left|B \cup B^{\prime}\right| \geq \frac{8}{15} n-\sigma$. Then again $B_{0}=B \cup B^{\prime}$, and by $(13)$

$$
\begin{aligned}
|N| \geq 0.4 n\left(2\left|B \cup B^{\prime}\right|+\left|C^{\prime}\right|-0.6 n\right) & \geq 0.4 n\left(\frac{16}{15} n-2 \sigma+\left|C^{\prime}\right|-0.6 n\right) \\
& =0.4 n\left(\frac{7}{15} n-2 \sigma+\left|C^{\prime}\right|\right) .
\end{aligned}
$$

Therefore

$0.4 n\left(\frac{7}{15} n-2 \sigma+\left|C^{\prime}\right|\right) \leq|N| \leq|M| \Delta_{1} \Delta_{2} \leq\left(0.2 n+5 \sigma+0.5\left|C^{\prime}\right|\right)(0.6 n-\sigma)$, which yields

$$
\frac{1}{15} n^{2}-3.6 n \sigma+5 \sigma^{2} \leq\left|C^{\prime}\right|(-0.5 \sigma-0.1 n) \leq 0 .
$$

Again, this inequality cannot hold for $0<\sigma \leq 0.004 n$, a contradiction to (3). 


\section{Proof of Lemma 2, Part I}

In this section, we prove (10). Let $t=\Delta_{1} \Delta_{2}-|A|-\left|A^{\prime}\right|-\left|C^{\prime}\right|+2.5 \sigma$. The following lemma gives bounds on $|B|$ and $\left|B \cup B^{\prime}\right|$ in terms of $t$.

Lemma 4. If $\left|B \cup B^{\prime}\right| \leq \frac{8}{15} n-\sigma$, then

(a) $|B| \geq 2.5 t+1.5 \sigma$, and

(b) $|B| \geq 2 t+\left|C^{\prime}\right|+0.5 \sigma$.

Furthermore, if $\left|C^{\prime}\right|<\frac{1}{15} n$, then

(c) $\left|B \cup B^{\prime}\right| \geq 2.5\left(t+\left|C^{\prime}\right|\right)$.

Proof. Recall that

$$
\begin{aligned}
t & =\Delta_{1} \Delta_{2}-|A|-\left|A^{\prime}\right|-\left|C^{\prime}\right|+2.5 \sigma \\
& \leq 0.6 n-\sigma-\left(n-\left|B \cup B^{\prime}\right|-\sigma\right)+2.5 \sigma \\
& \leq\left|B \cup B^{\prime}\right|-0.4 n+2.5 \sigma .
\end{aligned}
$$

Hence statement (a) of the lemma is true if the inequality $|B| \geq 2.5(\mid B \cup$ $\left.B^{\prime} \mid-0.4 n+2.5 \sigma\right)+1.5 \sigma$ holds. This is equivalent to

$$
0.4 n \geq \frac{3}{5}\left|B \cup B^{\prime}\right|+\frac{2}{5}\left|B^{\prime}\right|+3.1 \sigma .
$$

Since $\left|B \cup B^{\prime}\right| \leq \frac{8}{15} n-\sigma$ (and hence $\left|B^{\prime}\right| \leq \frac{2}{15} n-\sigma$, by (5)), the RHS of the last inequality is at most

$$
\frac{3}{5}\left(\frac{8}{15} n-\sigma\right)+\frac{2}{5}\left(\frac{2}{15} n-\sigma\right)+3.1 \sigma=\frac{28 n}{75}+2.1 \sigma .
$$

Thus to prove (a), it is sufficient to prove that

$$
2.1 \sigma \leq 0.4 n-\frac{112 n}{300}=\frac{8 n}{300},
$$

which is true when $\sigma \leq 0.004 n$. This proves (a).

Similarly, statement (b) of the lemma is true if the inequality $|B| \geq$ $2\left(\left|B \cup B^{\prime}\right|-0.4 n+2.5 \sigma\right)+\left|C^{\prime}\right|+0.5 \sigma$ holds. This is equivalent to

$$
0.8 n \geq\left|B \cup B^{\prime} \cup C^{\prime}\right|+\left|B^{\prime}\right|+5.5 \sigma .
$$

Since $\left|B \cup B^{\prime} \cup C^{\prime}\right| \leq 0.6 n-\sigma$ and $\left|B^{\prime}\right| \leq \frac{2}{15} n-\sigma$, we come to the inequality $0.8 n \geq \frac{11}{15} n+3.5 \sigma$ which holds if $\sigma \leq 0.004 n$. This proves (b).

Finally, (c) reduces to $\left|B \cup B^{\prime}\right| \geq 2.5\left(\left|B \cup B^{\prime}\right|-0.4 n+2.5 \sigma+\left|C^{\prime}\right|\right)$, which is equivalent to

$$
n \geq 1.5\left|B \cup B^{\prime} \cup C^{\prime}\right|+\left|C^{\prime}\right|+6.25 \sigma .
$$


As above $\left|B \cup B^{\prime} \cup C^{\prime}\right| \leq 0.6 n-\sigma$, and in addition we have $\left|C^{\prime}\right|<\frac{1}{15} n$. Thus we need

$$
n \geq 1.5(0.6 n-\sigma)+\frac{1}{15} n+6.25 \sigma,
$$

which is true for $\sigma \leq 0.004 n$.

\section{Proof of Statement (10) of Lemma 2.}

Suppose (10) does not hold. Choose $\Gamma \subset M \cap\left(B \cup B^{\prime}\right)$ with

$$
|\Gamma|=\left\{\begin{array}{l}
t+\frac{1}{2}\left|C^{\prime}\right|, \text { if }\left|B \cup B^{\prime}\right| \geq \frac{8}{15} n-\sigma ; \\
t, \text { otherwise. }
\end{array}\right.
$$

Let $\Gamma_{B}=\Gamma \cap B$ and $\Gamma_{B^{\prime}}=\Gamma \cap B^{\prime}$, and let $t_{B}=\left|\Gamma_{B}\right|$ and $t_{B^{\prime}}=\left|\Gamma_{B^{\prime}}\right|$.

For each $x \in \Gamma$, we choose $a(x) \in A$ and $b(x) \in B_{0}$ such that $x$ is the central vertex on the unique $(a, b ; 2,1)$-link. If $b(x) \in B^{\prime}$, then by the definition of $B^{\prime}$, there exists a $b_{1}(x) \in B$ such that $b(x) b_{1}(x) \notin E_{1}$ and there is no $\left(b(x), b_{1}(x) ; 2,1\right)$-link.

Define the auxiliary graph $\mathcal{B}$ as follows: $V(\mathcal{B})=B \cup B^{\prime}$ and $E(\mathcal{B})=$ $G_{\mathcal{B}} \cup D_{\mathcal{B}}$, where

$$
D_{\mathcal{B}}=\left\{\overrightarrow{x y}: x, y \in B \cup B^{\prime}, \text { there is an }(x, y ; 1,2) \text {-link }\right\}
$$

and

$$
\begin{array}{r}
G_{\mathcal{B}}=\left\{x y: x \in \Gamma, y \in B \cup B^{\prime}, x y \in E_{2} \text { or } a(x) y \in E_{2} \text { or } b(x) y \in E_{1}\right. \\
\text { or } \left.b_{1}(x) y \in E_{1}\right\},
\end{array}
$$

where $b_{1}(x) y \in E_{1}$ applies only when $b(x) \in B^{\prime}$.

We call edges in $G_{\mathcal{B}}$ and $D_{\mathcal{B}} G$-edges and $D$-edges, respectively.

We can bound the out-degrees of the vertices in $\mathcal{B}$ as follows.

Claim 4. (c1) The out-degree of each vertex in $B$ in $\mathcal{B}$ is at most

$$
d=\Delta_{1} \Delta_{2}-|A|-\left|A^{\prime}\right|-\left(\left|C^{\prime}\right|-\Delta_{1}\right)=t-2.5 \sigma+\Delta_{1} .
$$

(c2) The out-degree of each vertex in $B^{\prime}$ in $\mathcal{B}$ is at most

$$
d^{\prime}=\Delta_{1} \Delta_{2}-|A|-\left(\left|A^{\prime}\right|-\Delta_{1}\right)=t-2.5 \sigma+\Delta_{1}+\left|C^{\prime}\right| .
$$

Proof. For every vertex $u \in B \cup B^{\prime}$, the number of (1,2)-links starting from $u$ is at most $\Delta_{1} \Delta_{2}$, at least $|A|$ of those land in $A$ by Claims 2 and 3 .

To prove (c1), observe that by Claim 3 and the definition of $C^{\prime}$, at least $\left|A^{\prime}\right|+\left|C^{\prime}\right|-\Delta_{1}(1,2)$-links starting at $u$ land in $A^{\prime} \cup C^{\prime}$, thus at most $\Delta_{1} \Delta_{2}-$ $\left(|A|+\left|A^{\prime}\right|+\left|C^{\prime}\right|-\Delta_{1}\right)$ of those (1,2)-links land in $B \cup B^{\prime}$. 
By the definition of $B^{\prime}$, there exists $x \in B$ such that $u x \notin E_{1}$ and $\overrightarrow{x u} \notin D_{\mathcal{B}}$ when $u \in B^{\prime}$. To prove (c2), it suffices to show that for each $a^{\prime} \in A^{\prime}-N_{1}(x)$, there is a $\left(u, a^{\prime} ; 1,2\right)$-link.

Suppose not. Then by the definition of $A^{\prime}$, there is $a^{\prime \prime} \in A$ such that $a^{\prime} a^{\prime \prime} \notin E_{2}$ and there is no $\left(a^{\prime}, a^{\prime \prime} ; 1,2\right)$-link. Note that $a^{\prime} a^{\prime \prime} \notin E_{2}, a^{\prime} x \notin E_{1}$, $u x \notin E_{1}$ and $u^{*} a^{\prime \prime}, u^{*} x \notin E(G)$, thus by Lemma 1 , after a $\left(u^{*}, a^{\prime \prime}, a^{\prime}, u, x\right)$ swap, there is no conflicting edges, a contradiction.

Therefore, the number of $G$-edges incident with $B-\Gamma$ and $D$-edges (directed) from $B-\Gamma$ is at most $\left(|B|-t_{B}\right) d+2 \sigma|\Gamma|$, and the number of $G$-edges incident with $\left(B \cup B^{\prime}\right)-\Gamma$ and $D$-edges (directed) from $\left(B \cup B^{\prime}\right)-\Gamma$ is at most

$$
\left(|B|-t_{B}\right) d+\left(\left|B^{\prime}\right|-t_{B^{\prime}}\right) d^{\prime}+2 \sigma|\Gamma| .
$$

In the rest of the proof, we will prove that we have more $G$-edges or $D$-edges incident with $\left(B \cup B^{\prime}\right)-\Gamma$ than is given by (15). The obtained contradiction will prove the lemma. To do this, we will show that we may assign $|\Gamma|$ distinct $G$-edges or $D$-edges to each vertex in $\left(B \cup B^{\prime}\right)-\Gamma$.

If each $u \in\left(B \cup B^{\prime}\right)-\Gamma$ has either a $G$-edge or a $D$-edge to every vertex of $\Gamma$, we are done. Otherwise, consider each vertex $x \in\left(B \cup B^{\prime}\right)-\Gamma$ not having $|\Gamma|$ edges to $\Gamma$.

Let $\Gamma_{x}=\left\{y \in \Gamma: x y \notin G_{\mathcal{B}}, \overrightarrow{x y} \notin D_{\mathcal{B}}\right\}$. If $\Gamma_{x}=\emptyset$ then $x$ has $|\Gamma|$ edges to $\Gamma$.

For each vertex $y \in \Gamma_{x}$, let $Z_{y}=\left\{z \in\left(B \cup B^{\prime}\right)-\Gamma-\{x\}: y z \notin E, \overrightarrow{y z} \notin D_{\mathcal{B}}\right\}$.

Next, we prove two technical claims that will be helpful in the final counting argument.

Claim 5. Given $z \in Z_{y}$ such that $a(y) z \notin E_{2}$, and if $b(y) \in B^{\prime}$ implies that $b(y) z \notin E_{2}$ and $b_{1}(y) z \notin E_{1}$, then $\overrightarrow{z x} \in D_{\mathcal{B}}$.

Proof. Assume $\overrightarrow{z x} \notin D_{\mathcal{B}}$ for some $z \in Z_{y}$ satisfying the conditions in Claim 5 . Then $\overrightarrow{x y}, \overrightarrow{y z}, \overrightarrow{z x} \notin D_{\mathcal{B}}$ and $y z \notin E, x y \notin E_{2}$. By Lemma 1, a $(y, x, z)$-swap creates no new conflicting edges. Note then after the $(y, x, z)$-swap, there is no $(a(y), b(y) ; 2,1)$-link, since $x a(y), z a(y) \notin E_{2}$ and $x b(y) \notin E_{1}$.

If $b(y) \in B$, then since $\overrightarrow{a(y) u^{*}}, \overrightarrow{u^{*} b(y)}, \overrightarrow{b(y) a(y)} \notin D_{\mathcal{B}}$ and $a(y) u^{*}, b(y) u^{*} \notin$ $E(G)$, by Lemma 1 , an $\left(a(y), b(y), u^{*}\right)$-swap gives a packing of $G_{1}$ and $G_{2}$.

If $b(y) \in B^{\prime}$, then since $x b_{1}(y) \notin E_{1}, b(y) z \notin E_{2}$ and $b_{1}(y) z \notin E_{1}$, after the $(y, x, z)$-swap, there is still no $\left(b(y), b_{1}(y) ; 2,1\right)$-link. Therefore, after a $\left(u^{*}, a(y), b(y), b_{1}(y)\right)$-swap, by Lemma $1, G_{1}$ and $G_{2}$ pack.

Claim 6. For any subset $\Gamma_{x}^{\prime} \subseteq \Gamma_{x}$, there is some vertex $y_{0} \in \Gamma_{x}^{\prime}$ with the out-degree at least $0.5\left(\left|\Gamma_{x}^{\prime}\right|-2 \sigma\right)$ in $\mathcal{B}\left[\Gamma_{x}^{\prime}\right]$. 
Proof. Consider any vertex $y \in \Gamma_{x}^{\prime}$. We claim that for each $z \in \Gamma_{x}^{\prime}-N_{1}(b(y))-$ $N_{2}(a(y))-N_{1}\left(b_{1}(y)\right)-N_{2}(b(y))$, either $\overrightarrow{y z}$ or $\overrightarrow{z y}$ is a $D$-edge. Otherwise, since $z b(y) \notin E_{1}, z a(y) \notin E_{2}$, after a $(y, z)$-swap, there is no $(a(y), b(y) ; 2,1)$-link.

Then if $b(y) \in B$, after a $\left(u^{*}, a(y), b(y)\right)$-swap, by Lemma 1 , there are no conflicting edges anymore. This is a contradiction.

If $b(y) \in B^{\prime}$, since $z b(y) \notin E_{1}$ and $z b_{1}(y) \notin E_{2}$, after the $(y, z)$-swap, there is still no $\left(b(y), b_{1}(y) ; 2,1\right)$-link. Therefore, after an $\left(u^{*}, a(y), b(y), b_{1}(y)\right)$-swap, by Lemma $1, G_{1}$ and $G_{2}$ pack. This is also a contradiction.

We may assume $\left|\Gamma_{x}^{\prime}\right| \geq 2 \sigma$. Therefore, the sum of the out-degrees of vertices in $\Gamma_{x}^{\prime}$ is at least $0.5\left|\Gamma_{x}^{\prime}\right|\left(\left|\Gamma_{x}^{\prime}\right|-2 \sigma\right)$. Then there is some vertex $y_{0} \in \Gamma_{x}^{\prime}$ such that the out-degree of $y_{0}$ is at least $0.5\left(\left|\Gamma_{x}^{\prime}\right|-2 \sigma\right)$.

Now, we do the final computations, considering two cases.

Case 1. $\left|B \cup B^{\prime}\right| \geq \frac{8}{15} n-\sigma$. Then $\left|C^{\prime}\right| \leq \frac{1}{15} n$. By Lemma $4,\left|B \cup B^{\prime}\right| \geq$ $2.5\left(t+\left|C^{\prime}\right|\right)$.

Claim 7. $\Gamma_{x}=\emptyset$, that is, each $x \in\left(B \cup B^{\prime}\right)-\Gamma$ has $|\Gamma|$ edges to $\Gamma$.

Proof. Suppose that $\Gamma_{x} \neq \emptyset$. By Claim 6, choose $y \in \Gamma_{x}$ with the out-degree at least $0.5\left(\left|\Gamma_{x}\right|-2 \sigma\right)$ in $\mathcal{B}\left[\Gamma_{x}\right]$. Then $y$ has at most $d^{\prime}-0.5\left(\left|\Gamma_{x}\right|-2 \sigma\right) D$-edges to $\left(B \cup B^{\prime}\right)-\Gamma$. By Claim 5 , there are $\left|Z_{y}\right|-\Delta_{2}-\sigma D$-edges from $Z_{y}$ to $x$.

Note that $\left|Z_{y}\right| \geq\left|B \cup B^{\prime}\right|-|\Gamma|-\sigma-\left(d^{\prime}-0.5\left(\left|\Gamma_{x}\right|-2 \sigma\right)\right)$. To assign $|\Gamma|$ $D$-edges to $x$, it suffices to require

$$
\left|B \cup B^{\prime}\right|-|\Gamma|-\sigma-\left(d^{\prime}-0.5\left(\left|\Gamma_{x}\right|-2 \sigma\right)\right)-\Delta_{2}-\sigma \geq\left|\Gamma_{x}\right|,
$$

that is,

$$
\begin{aligned}
\left|B \cup B^{\prime}\right| & \geq|\Gamma|+d^{\prime}+\left|\Gamma_{x}\right|-0.5\left(\left|\Gamma_{x}\right|-2 \sigma\right)+2 \sigma+\Delta_{2} \\
& =t+d^{\prime}+0.5\left|\Gamma_{x}\right|+0.5\left|C^{\prime}\right|+\sigma+\Delta_{2} \\
& =2 t+0.5\left|\Gamma_{x}\right|+1.5\left|C^{\prime}\right|-0.5 \sigma .
\end{aligned}
$$

It suffices that

$$
\left|B \cup B^{\prime}\right| \geq 2.5 t+1.5\left|C^{\prime}\right|,
$$

and this is true since $\left|B \cup B^{\prime}\right| \geq 2.5\left(t+\left|C^{\prime}\right|\right)$.

Thus, in this case, we have assigned $|\Gamma|$ distinct $G$-edges or $D$-edges to each vertex in $\left(B \cup B^{\prime}\right)-\Gamma$.

Now, consider the edges incident with $\left(B \cup B^{\prime}\right)-\Gamma$. We have

$$
\begin{aligned}
& \left(\left|B \cup B^{\prime}\right|-|\Gamma|\right)|\Gamma| \leq\left(|B|-t_{B}\right) d+\left(\left|B^{\prime}\right|-t_{B^{\prime}}\right) d^{\prime}+2 \sigma|\Gamma| \\
& \quad \leq\left(|B|-t_{B}\right)\left(t-2.5 \sigma+\Delta_{1}\right)+\left(\left|B^{\prime}\right|-t_{B^{\prime}}\right)\left(t-2.5 \sigma+\left|C^{\prime}\right|+\Delta_{1}\right)+2 \sigma|\Gamma| \\
& \quad=\left(\left|B \cup B^{\prime}\right|-|\Gamma|\right)\left(t-2.5 \sigma+\Delta_{1}\right)+\left|C^{\prime}\right|\left(\left|B^{\prime}\right|-t_{B^{\prime}}\right)+2 \sigma|\Gamma| \\
& \quad \leq\left(\left|B \cup B^{\prime}\right|-|\Gamma|\right) t+\left|C^{\prime}\right|\left(\left|B^{\prime}\right|-t_{B^{\prime}}\right)-\left(\left|B \cup B^{\prime}\right|-|\Gamma|\right)(1.5 \sigma)+2 \sigma|\Gamma| .
\end{aligned}
$$


Since $\left|B \cup B^{\prime}\right|-|\Gamma| \geq 2.5\left(t+\left|C^{\prime}\right|\right)-\left(t+0.5\left|C^{\prime}\right|\right) \geq 1.5|\Gamma|$,

$$
-\left(\left|B \cup B^{\prime}\right|-|\Gamma|\right)(1.5 \sigma)+2 \sigma|\Gamma| \leq 0 .
$$

From the above inequality, we have

$$
\frac{1}{2}\left(\left|B \cup B^{\prime}\right|-|\Gamma|\right)\left|C^{\prime}\right| \leq\left|C^{\prime}\right|\left(\left|B^{\prime}\right|-t_{B^{\prime}}\right) .
$$

It follows that

$$
|B| \leq t_{B}+\left(\left|B^{\prime}\right|-t_{B^{\prime}}\right) \leq t+\left|B^{\prime}\right|
$$

By (14),

$$
t \leq\left|B \cup B^{\prime}\right|-0.4 n+2.5 \sigma \leq \Delta_{1} \Delta_{2}-0.4 n+2.5 \sigma \leq 0.2 n+1.5 \sigma .
$$

Thus (17) together with (6) implies

$$
0.4 n \leq(0.2 n+1.5 \sigma)+(0.2 n-2 \sigma),
$$

a contradiction.

Case 2. $\left|B \cup B^{\prime}\right| \leq \frac{8}{15} n-\sigma$. By Lemma $4,|B| \geq \max \left\{2.5 t+1.5 \sigma, 2 t+\left|C^{\prime}\right|+0.5 \sigma\right\}$.

Claim 8. $\Gamma_{x} \cap \Gamma_{B}=\emptyset$.

Proof. Let $t_{x}=\left|\Gamma_{x} \cap \Gamma_{B}\right|$ and assume $t_{x} \neq 0$. By Claim 6, choose $y_{1} \in \Gamma_{x} \cap \Gamma_{B}$ with the out-degree in $\mathcal{B}\left[\Gamma_{x} \cap \Gamma_{B}\right]$ at least $0.5\left(t_{x}-2 \sigma\right)$. Then $y_{1}$ has at most $d-0.5\left(t_{x}-2 \sigma\right) D$-edges to $\left(B \cup B^{\prime}\right)-\Gamma$. By Claim 5, there are $\left|Z_{y_{1}}\right|-\Delta_{2}-\sigma$ $D$-edges from $Z_{y_{1}}$ to $x$.

Note that

$$
\left|Z_{y_{1}}\right| \geq\left|B \cup B^{\prime}\right|-t-\sigma-\left(d-0.5\left(t_{x}-2 \sigma\right)\right) \geq|B|-t_{B}-\sigma-\left(d-0.5\left(t_{x}-2 \sigma\right)\right) .
$$

To assign $t D$-edges to $x$, it suffices to require

$$
|B|-t_{B}-\sigma-\left(d-0.5\left(t_{x}-2 \sigma\right)\right)-\Delta_{2}-\sigma \geq\left|\Gamma_{x}\right|=t_{x}+\left|\Gamma_{x} \cap B^{\prime}\right|,
$$

that is,

$$
|B| \geq 2 \sigma+d+\Delta_{2}+t_{B}+\left|\Gamma_{x} \cap B^{\prime}\right|+t_{x}-0.5\left(t_{x}-2 \sigma\right) .
$$

Since $t_{B}+\left|\Gamma_{x} \cap B^{\prime}\right| \leq t,(18)$ holds if

$$
|B| \geq 1.5 t+d+3 \sigma+\Delta_{2}=2.5 t+1.5 \sigma .
$$

Since $|B| \geq 2.5 t+1.5 \sigma$, we are done. 
Claim 9. $\Gamma_{x}=\emptyset$, that is, each $x \in\left(B \cup B^{\prime}\right)-\Gamma$ has $|\Gamma|$ edges to $\Gamma$.

Proof. Assume that $\Gamma_{x} \neq \emptyset$. By Claim 6 , there exists $y_{2} \in \Gamma_{x} \cap B^{\prime}$ with the out-degree at least $0.5\left(\left|\Gamma_{x} \cap B^{\prime}\right|-2 \sigma\right)$ in $\mathcal{B}\left[\Gamma_{x} \cap B^{\prime}\right]$. Then $y_{2}$ has at most $d^{\prime}-0.5\left(\left|\Gamma_{x} \cap B^{\prime}\right|-2 \sigma\right) D$-edges to $B-\Gamma$.

By Claim 5, there are $\left|Z_{y_{2}}\right|-\Delta_{2}-\sigma D$-edges from $Z_{y_{2}}$ to $x$.

Note that $\left|Z_{y_{2}}\right| \geq|B|-t_{B}-\sigma-\left(d^{\prime}-0.5\left(\left|\Gamma_{x} \cap B^{\prime}\right|-2 \sigma\right)\right)$. By Claim 8, to assign $t$ edges to $x$, it suffices to require

$$
\left|Z_{y_{2}}\right|-\Delta_{2}-\sigma \geq|B|-t_{B}-\sigma-\left(d^{\prime}-0.5\left(\left|\Gamma_{x} \cap B^{\prime}\right|-2 \sigma\right)\right)-\Delta_{2}-\sigma \geq t-t_{B}
$$

To get (19), it suffices that

$$
|B| \geq t+d^{\prime}+2 \sigma+\Delta_{2}=2 t+\left|C^{\prime}\right|+0.5 \sigma .
$$

Since $|B| \geq 2 t+\left|C^{\prime}\right|+0.5 \sigma$, the above inequality holds.

Thus, in this case, we have assigned $|\Gamma|$ distinct $G$-edges or $D$-edges to each vertex in $\left(B \cup B^{\prime}\right)-\Gamma$.

Now consider the edges incident with $B-\Gamma$. We have

$$
\left(|B|-t_{B}\right) t \leq\left(|B|-t_{B}\right) d+2 \sigma t,
$$

which yields $|B|<t_{B}+2 \sigma t / t-d$. Since $t-d \geq 2.5 \sigma-\Delta_{1} \geq 1.5 \sigma$ and

$$
|B| \geq 2.5 t \geq t_{B}+1.5 t \geq t_{B}+\frac{4 t}{3} \geq t_{B}+t \frac{2 \sigma}{t-d},
$$

we have a contradiction. This completes the proof of (10) in Lemma 2.

\section{Proof of Lemma 2, Part II}

We need to prove (11). The proof mostly repeats part of the previous section and is simpler, since by (8), $\left|A \cup A^{\prime}\right|$ is always less than $0.5 n$. But there is some asymmetry between $A$ and $B$. So we mostly will refer to Section 5 but will give definitions and prove a couple of claims to be on the safe side.

Let $t^{\prime}=\Delta_{1} \Delta_{2}-|B|-\left|B^{\prime}\right|-\left|C^{\prime}\right|+2.5 \sigma$. Symmetrically to Lemma 4, we have the following fact. 
Lemma $4^{\prime}$. (a) $|A| \geq 2.5 t^{\prime}+1.5 \sigma$, and (b) $|A| \geq 2 t^{\prime}+\left|C^{\prime}\right|+0.5 \sigma$.

The proof repeats that of Lemma 4 . Recall again that we do not need a statement symmetrical to (c) of Lemma 4, since $\left|A \cup A^{\prime}\right|<0.5 n$.

So, suppose that (11) does not hold. Choose $\Gamma \subset M \cap\left(A \cup A^{\prime}\right)$ with $|\Gamma|=t^{\prime}$. Let $\Gamma_{A}=\Gamma \cap A$ and $\Gamma_{A^{\prime}}=\Gamma \cap A^{\prime}$, and let $t_{A}=\left|\Gamma_{A}\right|$ and $t_{A^{\prime}}=\left|\Gamma_{A^{\prime}}\right|$.

For each $x \in \Gamma$, we choose $a(x) \in A, b(x) \in B_{0}$ such that $x$ is the central vertex lying on the unique $(a, b ; 2,1)$-link. If $b(x) \in B^{\prime}$, then by the definition of $B^{\prime}$, there exists $b_{1}(x) \in B$ such that $b(x) b_{1}(x) \notin E_{1}$ and there is no $\left(b(x), b_{1}(x) ; 2,1\right)$-link.

Define the auxiliary graph $\mathcal{A}$ as follows: $V(\mathcal{A})=A \cup A^{\prime}$ and $E(\mathcal{A})=$ $G_{\mathcal{A}} \cup D_{\mathcal{A}}$, where

$$
D_{\mathcal{A}}=\left\{\overrightarrow{x y}: x, y \in A \cup A^{\prime}, \text { there is }(x, y ; 2,1) \text {-link }\right\}
$$

and

$$
\begin{array}{r}
G_{\mathcal{A}}=\left\{x y: x \in \Gamma, y \in A \cup A^{\prime}, x y \in E_{2} \text { or } a(x) y \in E_{2} \text { or } b(x) y \in E_{1}\right. \\
\text { or } \left.b_{1}(x) y \in E_{1}\right\},
\end{array}
$$

where $b_{1}(x) y \in E_{1}$ applies only when $b(x) \in B^{\prime}$.

We call the edges in $G_{\mathcal{A}}$ and $D_{\mathcal{A}} G$-edges and $D$-edges, respectively. The following claim is proved exactly as Claim 4.

Claim $4^{\prime}$. (c1') The out-degree of each vertex in $A$ in $\mathcal{A}$ is at most

$$
d=\Delta_{1} \Delta_{2}-|B|-\left|B^{\prime}\right|-\left(\left|C^{\prime}\right|-\Delta_{2}\right)=t^{\prime}-2.5 \sigma+\Delta_{2} .
$$

$\left(\mathrm{c} 2^{\prime}\right)$ The out-degree of each vertex in $A^{\prime}$ in $\mathcal{A}$ is at most

$$
d^{\prime}=\Delta_{1} \Delta_{2}-|B|-\left(\left|B^{\prime}\right|-\Delta_{2}\right)=t^{\prime}-2.5 \sigma+\Delta_{2}+\left|C^{\prime}\right| .
$$

Therefore the number of $G$-edges incident with $A-\Gamma$ and $D$-edges (directed) from $A-\Gamma$ is at most $\left(|A|-t_{A}\right) d+2 \sigma|\Gamma|$.

We now show that we may assign $|\Gamma|\left(=t^{\prime}\right)$ distinct $G$-edges or $D$-edges to each vertex in $A-\Gamma$.

If each $u \in A-\Gamma$ has either a $G$-edge or a $D$-edge to every vertex of $\Gamma$, then we are done. Otherwise, consider each vertex $x \in A-\Gamma$ not having $t^{\prime}$ such edges to $\Gamma$.

Let $\Gamma_{x}=\left\{y \in \Gamma: x y \notin G_{\mathcal{A}}, \overrightarrow{x y} \notin D_{\mathcal{A}}\right\}$.

For each vertex $y \in \Gamma_{x}$, let $Z_{y}=\left\{z \in A \cup A^{\prime}-\Gamma-\{x\}: y z \notin E, \overrightarrow{y z} \notin D_{\mathcal{A}}\right\}$. 
Claim $5^{\prime}$. If $z \in Z_{y}$ such that $b(y) z \notin E_{1}$, then $\overrightarrow{z x} \in D_{\mathcal{A}}$.

Proof. Assume $\overrightarrow{z x} \notin D_{\mathcal{A}}$ for some $z \in Z_{y}$ with $b(y) z \notin E_{1}$. Then there is no $(x, y ; 2,1)$-link, no $(y, z ; 2,1)$-link, no $(z, x ; 2,1)$-link, and $y z \notin E, x y \notin E_{2}$. By Lemma 1 , a $(x, y, z)$-swap creates no new conflicting edges. Note that, after the $(x, y, z)$-swap, there is no $(a(y), b(y) ; 2,1)$-link, since by the definition of $G_{\mathcal{A}}, x a(y) \notin E_{2}$ and $x b(y) \notin E_{1}$, and by our assumption $b(y) z \notin E_{1}$. Recall that $G_{1}$ stays fixed while the embedding of $G_{2}$ changes under a swap operation.

If $b(y) \in B$, then since there is no $\left(u^{*}, a(y) ; 2,1\right)$-link, no $(a(y), b(y) ; 2,1)$ link, no $\left(b(y), u^{*} ; 2,1\right)-$ link, and $a(y) u^{*}, b(y) u^{*} \notin E(G)$, by Lemma 1 , a $\left(u^{*}\right.$, $a(y), b(y))$-swap gives a packing of $G_{1}$ and $G_{2}$, a contradiction.

If $b(y) \in B^{\prime}$, then since $y b(y) \notin E_{2}, b_{1}(y) x \notin E_{1}$ and $b(y) x \notin E_{2}$ (since there is no $(x, y ; 2,1)$-link), after the $(x, y, z)$-swap, there is still no $\left(b(y), b_{1}(y) ; 2,1\right)$ link. By Lemma $1, G_{1}$ and $G_{2}$ pack after an $\left(u^{*}, a(y), b(y), b_{1}(y)\right)$-swap, since there are no $(2,1)$-links between consecutive vertices, no edges from $u^{*}$ to any other vertices, and $b(y) b_{1}(y) \notin E_{1}$, a contradiction.

Claim $6^{\prime}$. For any subset $\Gamma_{x}^{\prime} \subseteq \Gamma_{x}$, there is some vertex $y_{0} \in \Gamma_{x}^{\prime}$ with the out-degree at least $0.5\left(\left|\Gamma_{x}^{\prime}\right|-2 \sigma\right)$ in $\mathcal{A}\left[\Gamma_{x}^{\prime}\right]$.

Proof. Consider any vertex $y \in \Gamma_{x}^{\prime}$. We claim that for each $z \in \Gamma_{x}^{\prime}-N_{1}(b(y))-$ $N_{2}(a(y))-N_{1}\left(b_{1}(y)\right)-N_{2}(b(y))$, either $\overrightarrow{y z}$ or $\overrightarrow{z y}$ is a $D$-edge.

If not, then after a $(y, z)$-swap, there is no $(a(y), b(y) ; 2,1)$-link, since $z b(y) \notin E_{1}$ and $z a(y) \notin E_{2}$.

If $b(y) \in B$, then as above, a $\left(u^{*}, a(y), b(y)\right)$-swap gives a packing of $G_{1}$ and $G_{2}$. This is a contradiction.

If $b(y) \in B^{\prime}$, then after the $(y, z)$-swap there is still no $\left(b(y), b_{1}(y) ; 2,1\right)$ link, since $z b(y) \notin E_{2}$ and $z b_{1}(y) \notin E_{1}$. Therefore, as above, the $\left(u^{*}, a(y), b(y)\right.$, $\left.b_{1}(y)\right)$-swap gives a packing of $G_{1}$ and $G_{2}$. This is a contradiction. The sum of the out-degrees of vertices in $\Gamma_{x}^{\prime}$ is at least $0.5\left|\Gamma_{x}^{\prime}\right|\left(\left|\Gamma_{x}^{\prime}\right|-2 \sigma\right)$. Then there is some vertex $y_{0} \in \Gamma_{x}^{\prime}$ such that the out-degree of $y_{0}$ is at least $0.5\left(\left|\Gamma_{x}^{\prime}\right|-2 \sigma\right)$ in $\Gamma_{x}^{\prime}$.

The rest of the proof simply repeats Case 2 of the main proof in Section 5 . Again, we do not need Case 1 because $\left|A \cup A^{\prime}\right|<0.5 n$.

Acknowledgment. We thank the referees for helpful comments.

\section{References}

[1] M. Aigner and S. Brandt: Embedding arbitrary graphs of maximum degree two, J. London Math. Soc. (2) 28 (1993), 39-51. 
[2] N. Alon and E. Fischer: 2-factors in dense graphs, Disc. Math. 152 (1996), 13-23.

[3] B. BollobÁs: Extremal Graph Theory, Academic Press, London-New York, (1978).

[4] B. Bollobás and S. E. Eldridge: Packing of graphs and applications to computational complexity, J. Comb. Theory Ser. B 25 (1978), 105-124.

[5] B. Bollobás, A. Kostochka and K. Nakprasit: Packing $d$-degenerate graphs, J. Comb. Theory Ser. B 98(1) (2008), 85-94.

[6] P. A. Catlin: Subgraphs of graphs I., Disc. Math. 10 (1974), 225-233.

[7] P. A. CAtLin: Embedding subgraphs and coloring graphs under extremal degree conditions, Ph.D. Thesis, Ohio State Univ., Columbus, (1976).

[8] B. Csaba, A. Shokoufandeh and E. Szemerédi: Proof of a conjecture of Bollobás and Eldridge for graphs of maximum degree three, Combinatorica 23(1) (2003), 3572.

[9] N. Eaton: A near packing of two graphs, J. Comb. Theory Ser. B 80 (2000), 98-103.

[10] A. Hajnal and E. Szemerédi: Proof of conjecture of Erdős, in: Combinatorial Theory and its Applications, Vol. II (P. Erdős, A. Rényi and V. T. Sós editors), NorthHolland, (1970), 601-623.

[11] T. R. JEnsen and B. TofT: Graph coloring problems, Wiley-Interscience, (1995).

[12] H. Kaul and A. KostochKa: Extremal graphs for a graph packing theorem of Sauer and Spencer, Combin. Probab. Comput. 16 (2007), 409-416.

[13] N. Sauer and J. Spencer: Edge disjoint placement of graphs, J. Combin. Theory Ser. B 25 (1978), 295-302.

[14] M. Wozniak: Packing of graphs, Dissertationes Math. 362 (1997), 78 pp.

[15] H. P. YAP: Packing of graphs - a survey, Disc. Math. 72 (1988), 395-404.

Hemanshu Kaul

Department of Applied Mathematics

Illinois Institute of Technology

10 West 32nd Street

Chicago, IL 60616

USA

kaul@math.iit.edu

\author{
Alexandr Kostochka \\ Department of Mathematics \\ University of Illinois \\ 1409 West Green Street \\ Urbana, IL 61801 \\ USA \\ and
}

Institute of Mathematics

Novosibirsk, Russia

kostochk@math . uiuc .edu

\section{Gexin $\mathrm{Yu}$}

Department of Mathematics

Vanderbilt University

1326 Stevenson Center

Nashville, TN 37240

USA

gexin.yu@vanderbilt. edu 